Preprints of the

Max Planck Institute for

Research on Collective Goods

Bonn 2008/21

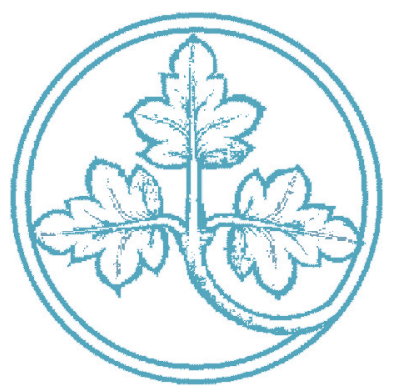

Apportionment, Fiscal Equalization and Decentralized Tax Enforcement

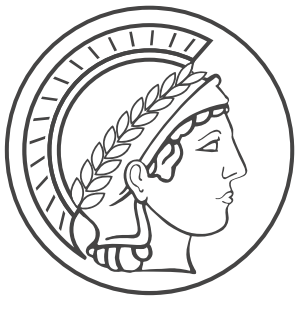




\title{
Apportionment, Fiscal Equalization and Decentralized Tax Enforcement
}

\author{
Christian Traxler / Andreas Reutter
}

April 2008 


\title{
Apportionment, Fiscal Equalization and Decentralized Tax Enforcement*
}

\author{
Christian Traxler ${ }^{\dagger} \quad$ Andreas Reutter ${ }^{\ddagger}$
}

April 2008

\begin{abstract}
We study tax evasion and decentralized tax enforcement in a federal economy with mobile capital and the endogenous formation of multiregional companies. Regions use their enforcement policy as a strategic instrument to engage in fiscal competition. Within this framework, we analyze the uncoordinated policy choice under formula apportionment (FA) and compare it to the incentives which derive from fiscal equalization (FE). As both systems redistribute collected revenues but not enforcement costs, they distort the regions' incentives to enforce taxes. At the same time, jurisdictions partially internalize the fiscal externalities caused by their enforcement policy. We show that the tradeoff between these two opposing effects differs between FA and FE, and crucially depends on the degree of interregional firm integration under FA. We discuss conditions under which FA, FE or a joint system of FA cum FE provides the 'best' incentives for decentralized tax enforcement.
\end{abstract}

Keywords: Tax Enforcement; Tax Evasion; Formula Apportionment; Fiscal Equalization; Tax Revenue Sharing.

JEL classification: $\mathrm{H} 77 ; \mathrm{H} 71 ; \mathrm{H} 26$.

${ }^{*}$ We would like to thank workshop participants in Konstanz, Munich, at the WZB Berlin, participants of the 63rd Conference of the IIPF (Warwick), as well as Ernesto Crivelli, Christoph Engel, Bernd Genser and, in particular, Andreas Haufler for helpful comments and suggestions. Financial support by the German Science Foundation (DFG), Priority Programme on Institutional Design of Federal Systems (Grant No. SPP 1142) is gratefully acknowledged.

${ }^{\dagger}$ Corresponding Author. Max Planck Institute for Research on Collective Goods. Kurt-Schumacher-Str. 10, D-53113 Bonn, Germany. Phone/Fax: +49 (0)228 91416-69/-62; E-mail: traxler@coll.mpg.de

${ }^{\ddagger}$ Department of Economics, University of Konstanz, Box D133, D-78457 Konstanz, Germany. 


\section{Introduction}

Over the last decades, a vast body of research has studied taxation within a federal system of governments (Oates, 1999). Thereby, surprisingly few contributions tackle an important practical aspect: the role of tax enforcement in a federal economy. Given the significant amount of taxes evaded and the equally impressive amount of resources spent on enforcing tax payments, ${ }^{1}$ this issue deserves more attention.

Apart from the highly decentralized system in Germany, where tax enforcement is under the sole responsibility of the Länder, many countries employ elements of de facto decentralized enforcement. This is the case for e.g. Canada, Japan, Spain, Switzerland, the UK as well as several developing and transitional economies, including China and Russia. ${ }^{2}$ Despite its widespread application, there are only few studies of decentralized enforcement. Cremer and Gahvari (2000) as well as Stöwhase and Traxler (2005) consider decentralized enforcement of taxes, taking into account evasion as well as an interregionally mobile tax base. Stricter enforcement then raises the effective taxation in a region, resulting in an outflow of the tax base, which corresponds to a positive externality. As long as the decentralized policy ignores this fiscal externality, enforcement measures will be inefficiently weak.

There is plenty of anecdotal evidence of cases where regions indeed seem to employ 'mild' enforcement as a strategic tool to attract a mobile tax base. In China there have been recent claims that '[s]ome regional governments openly abandoned law enforcement [...] to lure investors'. ${ }^{3}$ Similar reports come e.g. from Belgium (compare Cremer and Gahvari, 2000), Germany (see e.g. Senator fur Finanzen, 2005) and Russia (Libman and Feld, 2007).

Given that several countries may face institutional and political-economic constraints which render a (full) centralization of tax enforcement infeasible, ${ }^{4}$ it is important to ask, which policies can be used to improve the effectiveness of decentralized tax enforcement. A promising approach derives from the tax competition literature: as pointed out by Wildasin (1989), a system of Pigouvian subsidies can be used to internalize fiscal externalities from decentralized taxation. In practice, fiscal equalization (FE) schemes may fulfill this role (Smart, 1998; Köthenbürger, 2002). Stöwhase and Traxler (2005) show that FE makes regions internalize the externalities associated with tax enforcement. In addition, however, a FE system will typically cause an additional distortion: as long as only tax revenues are redistributed but enforcement costs are

\footnotetext{
${ }^{1}$ For the US, Slemrod (2007) estimates a tax gap of approximately $\$ 350$ billion in the year 2001 (more than 15 percent of the estimated actual tax liability). The annual budget of the Internal Revenue Service amounts to roughly $\$ 10$ billion (Sawicky, 2005).

${ }^{2}$ Mikesell (2003) and Martinez-Vazquez and Timofeev (2005) offer a comprehensive discussion of the different institutional arrangements.

${ }^{3}$ China Daily, July 23, 2007, p.4. Compare also Knight and Li (1999).

${ }^{4}$ Apart from externalities, there are of course several further arguments in favor (economies of scale) and against (information disadvantages) centralization. This suggest, that it is not immediate whether a centralization is indeed warranted or not. For a discussion of this issue, which is beyond the scope of the present paper, compare Martinez-Vazquez and Timofeev (2005).
} 
borne by the regions, there arises a disincentive for tax enforcement. ${ }^{5,6}$ Hence, there is a tradeoff between the internalization effect and the distortion from FE.

This paper contributes to the analysis of decentralized tax enforcement by exploring the incentives from another feature of many federal countries: formula apportionment (FA). While a growing amount of literature discusses the role of FA systems for profit shifting and tax competition, ${ }^{7}$ our focus is quite different: We study decentralized tax enforcement under FA and compare it to the equilibria which emerge under $\mathrm{FE}$, as well as under a joint system of fiscal equalization cum formula apportionment, as it is in place in Germany. We discuss the differences between these institutions and show, under which conditions which regime provides the 'best' incentives for decentralized auditing in a federal country.

Our analysis extends the model from Stöwhase and Traxler (2005) by incorporating the choice of firm as well as 'headquarter' (HQ) locations, similar to Black and Hoyt (1989). In addition to an endogenous evasion decision, the framework thereby captures the mobility of production factors as well as the endogenous emergence of multiregional companies. Allowing for two layers of mobility - mobility of capital and HQs - represents an innovative model feature which turns out to be crucial for the evaluation of a FA system. Moreover, it reflects that regions not only compete for production plants but also for HQs - just recall the impact of Boeing's announcement in 2001 that it will relocate its HQ from Seattle or compare one of the many governmental information campaigns for potential investors. A webpage supported by the Belgium Government, e.g., makes clear that 'Belgium highly values the presence of corporate headquarters and tends to actively pursue and attract them. ${ }^{18}$

Studying a stylized form of apportionment, we show that the incentives which derive from FA, crucially depend on the level of interregional company integration - a fact which has been so far neglected in the literature. Under FA, the taxes from multiregional companies are shared between jurisdictions according to a specific formula (e.g. based upon the factor input in the different regions). As sub-national governments bear the full costs of enforcement, FA then drives a wedge between the marginal costs and benefits of tax collection, distorting the efforts put into tax enforcement. At the same time, however, FA reduces the incentive to compete for multiregional companies, thereby working in favor of higher enforcement efforts. Hence, FA exerts opposing effects on decentralized tax enforcement, which are structurally similar to those from FE.

Compared to the case without any interregional redistribution of tax revenues, a FA system will result in more effective decentralized tax enforcement, if the level of interregional integration

\footnotetext{
${ }^{5}$ Compare Baretti et al. (2002), Büttner (2006), Dahlby and Warren (2003), Snoddon (2003) for empirical evidence on the (dis)incentives from FE.

${ }^{6}$ Stöwhase and Traxler (2005) further show that a scheme which redistributes revenues net of enforcement costs could in principle eliminate this disincentive. In practice, however, asymmetric information between different layers of government is likely to render such a mechanism infeasible.

${ }^{7}$ See, among many others, Eggert and Schjelderup (2003), Pethig and Wagener (2007), Riedel and Runkel (2007).

${ }^{8}$ See http://invest.belgium.be/en/headquarters. Several cases where companies relocated their headquarters are discussed e.g. in Klier and Testa (2002), Black and Hoyt (1989, p.1249). Compare also The Economist, March 10 2007, p.68.
} 
of firms is sufficiently low. Under the same conditions, however, a FE system might result in weaker enforcement. This difference stems from the fact that under FA only the revenues from multiregional companies are redistributed whereas under FE tax revenues from all companies are shared. In an economy with few multiregional companies, the distortion from FA is thus smaller than the one from FE. At the same time, however, the internalization effect from FA only partially depends on the degree of interregional integration. Hence, with a sufficiently small share of multiregional companies, the internalization will dominate the distortion under FA, but not necessarily under FE.

A similar result is found in the analysis of a joint FA and FE scheme. Adding FA to an existing FE system will improve the effectiveness of decentralized enforcement whenever interregional integration is sufficiently small. As both apportionment and equalization have slightly different purposes beyond providing incentives for decentralized policy choice, this finding is important. It shows that, apart from an eventually infeasible replacement of one by the other, the two systems can also be combined and still work as an attractive policy tool which provides regions with incentives to exert viable tax collection efforts.

The remainder of the paper is organized as follows. In the next section, we present the basic model. Section 3 discusses the decentralized choice of tax enforcement in the case without any interregional redistribution of tax revenues. Thereafter, we analyze the impact from FA (Section 4), FE (section 5) and a joint system of FA and FE (section 6). Policy implications and avenues for future research are discussed in the conclusion.

\section{The Model}

Our analysis extends the model of tax evasion within a federal economy from Stöwhase and Traxler (2005), allowing for a multiregional firm structure. Firms consist of two parts: a place of production and a 'headquarter' (HQ). While the location of production - in the following called the 'home region' of a firm - is exogenously given, the firms can either maintain their HQ at home or relocate it 'abroad'. In addition, capital input - and therewith the level of production - is mobile between regions. Independent from the HQ location, firms have to pay a unit tax on capital inputs. Motivated by the situation in Germany, we consider the tax rate as exogenously given and harmonized. The two parts of the firm are treated as one taxable unit, where taxes are collected at the HQs. ${ }^{9}$ This last assumption also shows that the model depicts sub- rather then international fiscal competition.

Firms may evade taxes, responding to the strictness of tax enforcement - expressed by the likeliness of facing an audit. Audits only take place at the firms' HQs and auditing rates may differ between regions. By choosing the location of their HQ, firms can therefore determine which regions' tax enforcement regime they will face.

The sequence of decisions is as follows: First, regions simultaneously choose (and credibly commit to) an audit rate. Thereafter, firms decide on their HQ location, capital inputs and the

\footnotetext{
${ }^{9}$ The production unit in our model is not a subsidiary company but only a dependent branch within a firm.
} 
level of tax evasion. Finally, audits take place and taxes are collected. We now describe the model's structure in more detail.

Federal Economy and Firm Structure. We consider an economy with two perfectly symmetric regions. In each region a continuum of firms with unit mass uses perfectly mobile capital and a fixed, immobile factor to produce a homogenous private good. All firms in the economy use the same technology, characterized by a strictly concave production function $f\left(k_{i \ell}\right)$, where $k_{i \ell}$ denotes the capital employed by a firm with production in region $i$ and a HQ in $\ell \in\{i, j\}$. In the following, the double subindexes $i i$ respectively $i j$ denote 'regional' respectively 'multiregional' firms, where the latter have their HQ abroad.

Firms can evade taxes by concealing a share $e_{i \ell}$ of the capital employed. Hiding inputs from tax authorities is costly. Following Cremer and Gahvari (1993), we assume that the costs are convex in $e_{i \ell}$ and linear in the tax base. Regional auditing policies are non-discriminatory, i.e. the jurisdictions audit regional ('home') and multiregional ('foreign') firms with the same probability $p_{\ell}{ }^{10}$ In case of an audit, the evasion is detected and the firm has to pay the full taxes plus a fine which is proportional to the taxes evaded. With probability $1-p_{\ell}$ the firm gets away with the evasion and only pays taxes on the declared amount of capital. The expected profit of a firm is then given by

$$
\pi_{i \ell}=f\left(k_{i \ell}\right)-r k_{i \ell}-g\left(e_{i \ell}\right) k_{i \ell}-p_{\ell}\left(t k_{i \ell}+(s-1) t e_{i \ell} k_{i \ell}\right)-\left(1-p_{\ell}\right) t\left(1-e_{i \ell}\right) k_{i \ell}
$$

where $r$ denotes the factor price for capital in the economy and the evasion costs are $g\left(e_{i \ell}\right) k_{i \ell}$ with $g^{\prime}>0$ and $g^{\prime \prime}>0$. Note again that the tax $(t)$ as well as the penalty rate $(s>1)$ are harmonized for both regions, whereas the auditing probability $p_{\ell}$ is not. Simplifying yields

$$
\pi_{i \ell}=f\left(k_{i \ell}\right)-\left(r+g\left(e_{i \ell}\right)+\tau_{i \ell}\right) k_{i \ell}
$$

with $\tau_{i \ell} \equiv t\left(1-e_{i \ell}+e_{i \ell} p_{\ell} s\right)$, the expected or effective tax rate faced by a firm with a HQ in $\ell$. This points out a central property of this model: Despite a harmonized tax rate, regions can determine the effective taxation by choosing their auditing rate $p_{\ell}$.

Taking the auditing policy as given, risk neutral firms choose $k_{i \ell}$ and $e_{i \ell}$ to maximize expected profits from (1). Optimal choices are characterized by the first order conditions

$$
\begin{aligned}
& g^{\prime}\left(e_{i \ell}\right)=\left(1-p_{\ell} s\right) t \\
& f^{\prime}\left(k_{i \ell}\right)=r+g\left(e_{i \ell}\right)+\tau_{i \ell} .
\end{aligned}
$$

Assuming $p_{\ell} s<1$, the optimal level of evasion is always positive. Several properties of this

\footnotetext{
${ }^{10}$ At this point, one could generalize the model in several aspects. First, we could consider an evasion technology which accounts for the fact that multiregional firms may face better opportunities - and hence lower costs - to evade taxes. Furthermore, we could allow for a different effectiveness (or costs) of auditing regional versus multiregional firms, resulting in a discriminatory audit policy. While the first extension would only quantitatively affect the HQ location choice (see (5) below), the second one would change the structure our analysis: Although the regions' strategic incentives to engage in fiscal competition would remain the same, the regimes discussed in sections 4 and 5 would have a different impact on the choice of a discriminatory enforcement policy. A formal analysis of a discriminatory policy choice is left for future research.
} 
framework derive from (2) and (3) (see Appendix A): First, firms will conceal more if the statutory tax rate increases or if the detection probability decreases - a standard result in the literature on evasion by firms (see e.g. Cremer and Gahvari, 1993). Second, an increase in the audit rate will raise (per unit) capital costs and hence decrease capital demand. This triggers an effect which is analogous to the impact of a tax increase on mobile capital in standard tax competition models. Third, each firm's optimal choice of capital input and tax evasion depends only on the location of its HQ - and not on its home region. This is the case since it is only the HQ location which determines the non-discriminatory auditing regime and thereby the effective tax rate for the firm. As firms are assumed to be identical with respect to evasion and production technologies, we get

$$
\pi_{i \ell}=\pi_{j \ell}=\pi_{\ell}, \quad e_{i \ell}=e_{j \ell}=e_{\ell}, \quad k_{i \ell}=k_{j \ell}=k_{\ell}, \quad \tau_{i \ell}=\tau_{j \ell}=\tau_{\ell} .
$$

In the optimum, firms with a HQ in $\ell$ will make the same profits, choose the same level of capital inputs respectively concealed capital, and will face the same effective tax rate $\tau_{\ell}$, irrespective of their home region. In the remainder of the paper, a single subindex of $\tau$ will therefore denote the effective tax rate in the region where a firm has its HQ.

Headquarter Location. We now turn to the firms' choice of the HQ location. A firm from region $i$ compares the expected profits if it maintains its $\mathrm{HQ}$ at home with the alternative of becoming a multiregional firm by relocating the HQ into region $j$. This comparison is influenced by the regions' effective tax rates (which are determined by the regional auditing policies) as well as $\underline{\theta} \leq \theta_{i} \leq \bar{\theta}$, some firm-specific net-benefits $(\theta>0)$ respectively net-costs $(\theta<0)$ from relocating the HQ abroad (compare Black and Hoyt, 1989). The parameter therefore captures the firms' costs from relocating the HQ as well as potential benefits from positioning the HQ in a different region (e.g. close to an important market). ${ }^{11}$

A firm from region $i$ will maintain its $\mathrm{HQ}$ at home iff $\theta \leq \hat{\theta}_{i}$, with

$$
\hat{\theta}_{i} \equiv \pi_{i i}-\pi_{i j}
$$

The threshold equals the gains (or losses) from maintaining the HQ at home instead of going abroad. All firms with costs above (respectively benefits below) this threshold will keep their HQ in $i$, while those with $\theta>\hat{\theta}_{i}$ will relocate it abroad.

Let the distribution of $\theta$ among firms in both regions be given by the c.d.f. $H(\theta)$, where the density function satisfies $0<h(\theta)<\infty$ on the interval $[\underline{\theta}, \bar{\theta}]$. The share of firms from region $i$ which maintain their HQ at home is then given by

$$
\sigma_{i}=H\left(\hat{\theta}_{i}\right)
$$

and the share of multiregional firms equals $1-\sigma_{i}$. One can interpret $\sigma_{i}$ as an indicator for the interregional integration of firms, where a high [low] level of $\sigma_{i}$ captures low [high] integration.

\footnotetext{
${ }^{11}$ See e.g. Klier and Testa (2002), Black and Hoyt (1989).
} 
Capital Market. In each region a representative household is endowed with a given amount of capital $\bar{k}_{i}$ (as well as one unit of the immobile factor). Hence, capital supply is exogenously fixed and market clearing requires

$$
\sum \bar{k}_{i}=\sum \tilde{k}_{i}
$$

where $\tilde{k}_{i} \equiv \sigma_{i} k_{i i}+\left(1-\sigma_{j}\right) k_{j i}$ denotes the (true) tax base of region $i$. It is composed of the capital from $\sigma_{i}$ regional firms as well as the capital from $1-\sigma_{j}$ multiregional (foreign) firms, which have relocated their HQs into $i{ }^{12}$

\section{Decentralized Tax Enforcement}

We will now study the endogenous choice of auditing rates. As a benchmark scenario, we first analyze the case of decentralized tax enforcement without any interregional sharing of tax revenues. Regional governments choose their enforcement efforts, taking into account the impact on tax evasion, the effective tax rate as well as the HQ and capital allocation. Authorities are assumed to maximize net revenues, consisting of taxes and penalties collected minus auditing costs. The costs, which are necessary to establish an auditing rate $p_{i}$ for a tax base $\tilde{k}_{i}$, are given by $c\left(p_{i}, \tilde{k}_{i}\right)$, with $c_{1} \geq 0, c_{11} \geq 0, c_{2} \geq 0, c_{22} \geq 0$ and $c_{12} \leq 0 .{ }^{13}$ Regional net revenue can be written as

$$
R_{i}=\tau_{i} \tilde{k}_{i}-c\left(p_{i}, \tilde{k}_{i}\right)
$$

with $\tau_{i}$ denoting the effective tax rate as defined above. Taking the other region's auditing rate as given, each authority then chooses $p_{i}$ in order to maximize $R_{i}$. The first order condition to this problem is given by

$$
\tilde{k}_{i} \frac{\partial \tau_{i}}{\partial p_{i}}+\tau_{i} \frac{\partial \tilde{k}_{i}}{\partial p_{i}}=C_{i}^{\prime}
$$

with

$$
C_{i}^{\prime} \equiv c_{1}+c_{2} \frac{\partial \tilde{k}_{i}}{\partial p_{i}}
$$

and $\partial \tau_{i} / \partial p_{i}>0$ respectively $\partial \tilde{k}_{i} / \partial p_{i}<0$ are derived in Appendix A. ${ }^{14}$ Condition (8) characterizes the regions' reaction functions which, in turn, determine the Nash equilibrium of the decentralized policy choice.

Let us now compare the result from decentralized auditing with the outcome under a centralized system. Consider a central authority which chooses (regional) tax enforcement levels in order to maximize total revenues $\max R \equiv \sum R_{i}$. The revenue maximizing choice is then

\footnotetext{
12 Equivalently, we can define $K_{i} \equiv \sigma_{i} k_{i i}+\left(1-\sigma_{i}\right) k_{i j}$, capturing the capital employed in the production process of jurisdiction $i$. It is straightforward that there holds $\sum K_{i}=\sum \tilde{k}_{i}$ in equilibrium. $K_{i}$ consists of the capital from $\sigma_{i}$ regional firms as well as from $1-\sigma_{i}$ multiregional (home) firms, which have their HQ in $j$. This shows that we treat the HQ as a 'letterbox', which does not employ any capital. We will come back to this point in the following.

${ }^{13}$ The subindexes here denote the derivative(s) of $c(.,$.$) w.r.t. the first respectively second argument(s).$

${ }^{14}$ It is straightforward to show that the second order condition to this problem holds under our assumptions on $c(.,$.$) and due to the concavity of \tau_{i}$ in $p_{i}$.
} 
characterized by

$$
\tilde{k}_{i} \frac{\partial \tau_{i}}{\partial p_{i}}=c_{1}\left(p_{i}, \tilde{k}_{i}\right)
$$

(equivalently for both regions). ${ }^{15}$ Comparing this condition with (8), one can easily see the difference between the coordinated and the uncoordinated policy choice. Under decentralized tax enforcement both regions take into account the capital outflows caused by stricter auditing, which reduce the marginal benefits from auditing. In contrast, a central authority considers that capital outflows from one region correspond to capital inflows - and ceteris paribus higher tax revenues - in the other jurisdiction. Stated differently, the central authority incorporates the fiscal externalities associated with regional auditing policies. The marginal benefits considered in the centralized decision - and therewith the chosen auditing rates - are therefore higher.

Proposition 1 The decentralized policy choice results in auditing rates which are below the level a central authority would implement.

Proof. See Appendix.

This result is analogous to the findings in Cremer and Gahvari (2000) and Stöwhase and Traxler (2005). ${ }^{16}$ The proposition corresponds to the standard case of an inefficiently low taxation of mobile capital in the tax competition literature (Wilson, 1986; Zodrow and Mieszkowski, 1986). Although statutory tax rates are harmonized, regions use audit rates as strategic tools to engage in fiscal competition. Decentralized tax enforcement then results in an 'inefficiently' low level of auditing, respectively an 'inefficiently' high level of tax evasion - inefficient, from the perspective of revenue maximization.

In the following, we study how this result changes once we introduce different forms of tax sharing into our analysis. Before we do so, let us remark that we can not interpret Proposition 1 as a clear-cut result in favor of a centralized auditing system. (The same remark applies to the results derived in section 6.2 below.) Our framework is tailored to the analysis of a decentralized policy choice under different forms of interregional redistribution. A proper comparison of centralized and decentralized tax enforcement calls for including several arguments (such as economies of scale in auditing, information (dis)advantages, etc.) which are neglected here, as they would blur the focus of our study.

\section{Decentralized Enforcement under Apportionment}

We now introduce a stylized form of formula apportionment (FA) into the model. We assume that a share $0<\alpha<1$ of tax revenues collected from multiregional firms is transferred back into the firm's home region - i.e. from the region of the HQ into the region where production takes place. While in practice the value of $\alpha$ depends on a specific apportionment formula (e.g. based

\footnotetext{
${ }^{15}$ Here we made use of $\partial \tilde{k}_{i} / \partial p_{i}+\partial \tilde{k}_{j} / \partial p_{i}=0$ as well as (4) and the properties of a symmetric equilibrium: $p_{i}=p_{j} \Rightarrow \tau_{i}=\tau_{j}$ and $c_{2}\left(p_{i}, \tilde{k}_{i}\right)=c_{2}\left(p_{j}, \tilde{k}_{j}\right)$ where $j$ denotes a region $j \neq i$.

${ }^{16}$ For $c(p, k)$ with $c_{22}=0$ Stöwhase and Traxler (2005) discuss that this basic result generalizes for $n \geq 2$ asymmetric regions.
} 
upon the payroll in the different regions), we consider an exogenous level of apportionment. Although this assumption is untypical, it is not crucial for our results. Note first, that all our main findings are independent of the level of $\alpha$, as we do not allow for income effects. Second, in contrast to most other studies of FA (e.g. Riedel and Runkel, 2007), multiregional firms have no incentive to shift profits or costs. This is due to the fact that statutory taxes are harmonized and that effective taxation is determined exclusively by the HQ location. Instead of modeling HQs as 'letterboxes' (compare footnote 12) we could thus introduce an endogenous level of inputs employed in HQs and base the apportionment formula upon e.g. the payroll or the inputs used by the two units of multiregional firms. ${ }^{17}$ Such an endogenous factor $\alpha$ would not qualitatively affect the strategic incentives for the regions, nor would the apportionment rule affect the factor allocation within multiregional firms. ${ }^{18}$

Under FA the net revenue of a region $i$ becomes

$$
R_{i}^{F A}=\sigma_{i} k_{i i} \tau_{i}+\alpha\left(1-\sigma_{i}\right) k_{i j} \tau_{j}+(1-\alpha)\left(1-\sigma_{j}\right) k_{j i} \tau_{i}-c\left(p_{i}, \tilde{k}_{i}\right)
$$

The first term on the RHS depicts the revenues collected from the fraction $\sigma_{i}$ of regional firms from $i$. The transfers under FA are reflected in the second and third term: The second term captures the fraction $\alpha$ of taxes collected from domestic multiregional firms (from $i$ with a HQ in $j$ ), which are transferred back into region $i$. The third term indicates taxes paid by foreign multiregional firms (from $j$ with a HQ in $i$ ). Since a share $\alpha$ of these revenues is transferred to the firms' home region $j$, only the fraction $1-\alpha$ remains in $i$.

In the case of decentralized tax enforcement a regional planner now chooses $p_{i}$ which maximizes $R_{i}^{F A}$. The first order condition to this problem is ${ }^{19}$

$$
\left(1-\alpha\left(1-\sigma_{i}\right)\right)\left(\tau_{i} \frac{\partial k_{i}}{\partial p_{i}}+k_{i} \frac{\partial \tau_{i}}{\partial p_{i}}\right)-\alpha\left(1-\sigma_{i}\right) \tau_{i} \frac{\partial k_{i}}{\partial p_{i}}+(1-\alpha) 2 k_{i} \tau_{i} \frac{\partial \sigma_{i}}{\partial p_{i}}=C_{i}^{\prime}
$$

From this condition one can identify several incentives which are embedded in the apportionment system:

A. Distortion. The first term on the LHS of (12) shows that FA reduces the regions' marginal benefits from auditing. While a region $i$ bears the full costs of auditing, it only receives a fraction $(1-\alpha)$ of the taxes enforced from foreign firms. This distortion clearly provides a disincentive for auditing.

B. Mitigation of $k$-Competition. The apportionment system reduces the incentives to engage in the competition for mobile capital. Under FA, revenues from domestic firms which

\footnotetext{
${ }^{17}$ One caveat is that our stylized modeling of FA does not capture a formula which is linked to a company's sales in the different regions (or to any other variable which is unaffected by the HQ location).

${ }^{18}$ Assume that the apportionment formula is based upon the capital allocation between the place of production and the HQ. In this case - and allowing for different evasion technologies at the two units - a variation in the auditing rate could result in a disproportionate change in the level of capital concealed at the HQ respectively at the production place. Thereby, the apportionment factor would change, i.e. $\alpha$ would become a function of $p_{i}$. It is easy to show, that including $\alpha\left(p_{i}\right)$ in (11) below, does only quantitatively change the subsequent first order condition in (12), as the term $\partial \alpha\left(p_{i}\right) / \partial p_{i}$ vanishes in a symmetric equilibrium between symmetric regions.

${ }^{19}$ Compare the Proof of Proposition 2 in the Appendix. There we also show $\partial \sigma_{i} / \partial p_{i}<0$.
} 
are taxed abroad are (partially) retrieved, whereas revenues from foreign multiregional firms are (partially) transferred abroad. This induces regions to internalize - at least to some extent - the fiscal externalities from its auditing policies. ${ }^{20}$ The effect, captured by condition (12)'s second term, therefore works in favor of higher auditing rates.

C. Mitigation of HQ-Competition. The third term on the LHS of (12) reflects a similar effect: FA reduces the revenue gains from attracting foreign HQs as well as the losses from loosing domestic HQs. This internalization effect mitigates the incentives to compete for HQs and thereby tends to increase auditing rates.

As compared to the benchmark case without any interregional redistribution of taxes, FA exerts incentives to reduce (effect A) as well as incentives to intensify (effects B and C) auditing. Hence, the overall impact of the apportionment system on the decentralized policy choice is in general ambiguous. Comparing conditions (12) and (8) delivers the following result:

Proposition 2 Under FA, the decentralized policy choice results in auditing rates which are below the level chosen in the absence of any tax sharing scheme iff

(i) $\hat{\lambda}_{i}>0$ and $\sigma_{i}<\hat{\mu}_{i}$.

The uncoordinated auditing rates under FA are above the level chosen in the absence of any tax sharing iff

(ii) $\hat{\lambda}_{i}>0$ and $\sigma_{i}>\hat{\mu}_{i}$, or (iii) $\hat{\lambda}_{i}<0$

with

$$
\hat{\lambda}_{i} \equiv k_{i} \frac{\partial \tau_{i}}{\partial p_{i}}+2 \tau_{i} \frac{\partial k_{i}}{\partial p_{i}} \quad \text { and } \quad \hat{\mu}_{i} \equiv 1+2 k_{i} \tau_{i} \frac{\partial \sigma_{i}}{\partial p_{i}} \frac{1}{\hat{\lambda}_{i}}
$$

Proof. See Appendix.

The proposition defines conditions under which the introduction of FA will either raise or lower the intensity of decentralized tax enforcement. The crucial thresholds in these conditions are $\hat{\lambda}_{i}$, which captures the sum of effects $\mathrm{A}$ and $\mathrm{B}$ discussed above, as well as $\hat{\mu}_{i}$, which measures the strength of effect $\mathrm{C}$ relative to $\hat{\lambda}_{i}$. If $\hat{\lambda}_{i}<0$, FA provides an unambiguous incentive to intensify auditing. This is the case whenever the marginal capital mobility, $\left|\partial k_{i} / \partial p_{i}\right|$, is relatively high and the impact of stricter auditing on the effective tax rate, $\partial \tau_{i} / \partial p_{i}$, is relatively small. For this scenario, there exists a strong incentive to compete for mobile capital. The mitigation of capital competition (effect B) then has a strong impact on the choice of auditing rates and dominates the distortion from FA (effect A), which is weaker for small $\partial \tau_{i} / \partial p_{i}$.

If marginal capital outflows are relatively small and the impact of auditing on effective taxation is relatively high, we will get $\hat{\lambda}_{i}>0$, i.e. the case where the distortion (A) dominates the mitigation effect B. However, FA also alleviates the competition for mobile HQs (effect $\mathrm{C}$

\footnotetext{
${ }^{20}$ Hence, the effect is similar - though not identical - to the internalization effects from revenue sharing discussed in Köthenbürger (2002) as well as in Stöwhase and Traxler (2005).
} 
from above), thereby providing a further incentive to raise auditing rates. As long as $\sigma_{i}>\hat{\mu}_{i}$, this last effect is sufficiently strong to outbalance (together with B) the distortion.

The intuition behind the last condition is straightforward. On the one hand, FA has little impact on a region's tax revenues for high levels of $\sigma_{i}$ (when there are only few multiregional firms). For a low degree of interregional integration the distortionary effect as well as the competition mitigating effect $\mathrm{B}$ are both relatively weak. ${ }^{21}$ On the other hand, effect $\mathrm{C}$ crucially depends on the marginal HQ-mobility but not on the absolute level of $\sigma_{i}$. The more sensitive HQ locations react to an increase in auditing, the more important becomes the apportionment's incentive to mitigate the HQ-competition. Stated more technically: the higher $\left|\partial \sigma_{i} / \partial p_{i}\right|$, the smaller $\hat{\mu}_{i}$ and the more likely condition $\sigma_{i}>\hat{\mu}_{i}$ will be met.

Following the same line of reasoning it is clear that for $\sigma_{i}<\hat{\mu}_{i}$ (and $\hat{\lambda}_{i}>0$ ) FA tends to further push auditing efforts below the level chosen in the absence of any tax sharing. This condition is more likely to be fulfilled, if there are many multiregional firms ( $\sigma_{i}$ is low) or if marginal $\mathrm{HQ}-$ mobility is small. Under these conditions, the mitigation effect $\mathrm{C}$ has only a minor impact and the distortion from FA dominates.

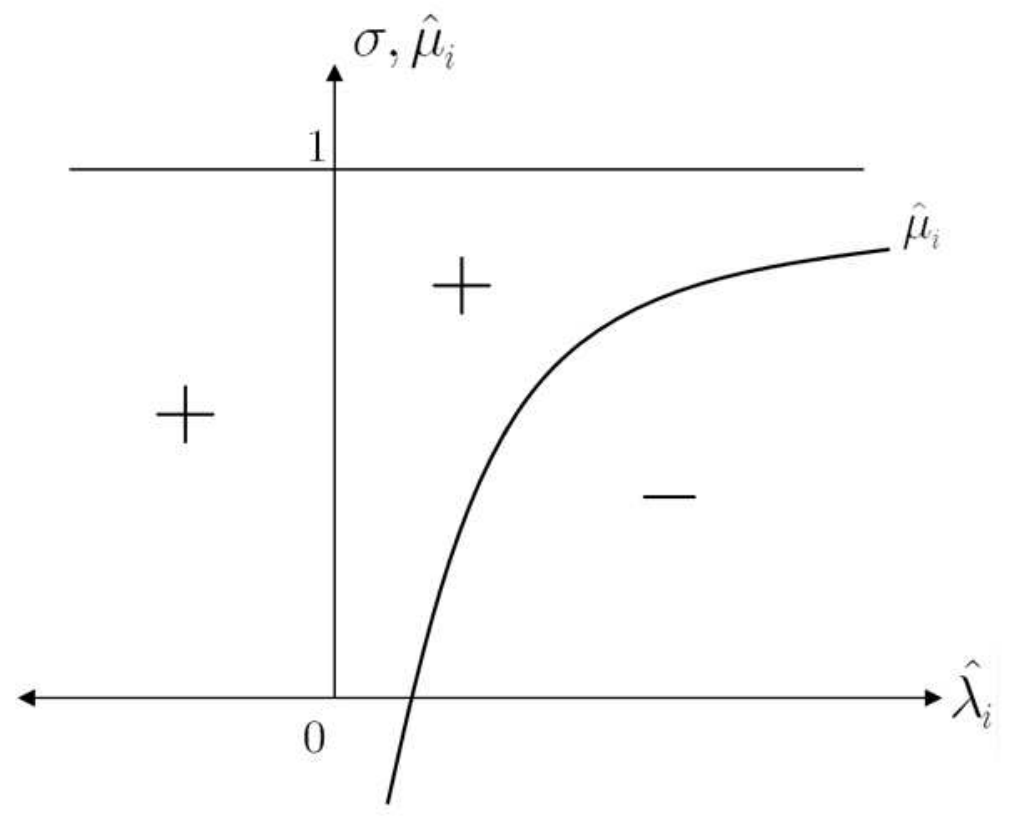

Figure 1: Impact of the Apportionment System

A graphical illustration of the three different cases from Proposition 2 is provided in Figure 1. The graph shows $\hat{\lambda}_{i}$ on the horizontal and $\sigma$ as well as $\hat{\mu}_{i}$ on the vertical axis. As long as $\hat{\lambda}_{i}<0$, the introduction of a FA system will unambiguously result in higher auditing rates for the whole range of $\sigma \in[0,1]$. For $\hat{\lambda}_{i}>0$, apportionment works in favor of higher auditing efforts only if $\sigma$ is above the $\hat{\mu}_{i}$-curve. In this case, the two mitigation effects $\mathrm{B}$ and $\mathrm{C}$ outbalances the disincentive for auditing (A). If, however, $\sigma_{i}<\hat{\mu}_{i}$, the distortion dominates. Note that an increase in $\hat{\lambda}_{i}$ captures that the distortion becomes stronger relative to effect B (e.g. if either capital mobility

\footnotetext{
${ }^{21}$ One can easily see from (12) that effect $\mathrm{A}$ as well as effect B are decreasing in $\sigma_{i}$.
} 
decreases or if the marginal impact of auditing on the effective tax rate increases). The higher $\hat{\lambda}_{i}$, the bigger the mitigation effect $\mathrm{C}$ must therefore be in order to outbalance the distortion. This is reflected by the threshold $\hat{\mu}_{i}$ which increases in $\hat{\lambda}_{i}$.

\section{Decentralized Enforcement under Fiscal Equalization}

We now turn to a different form of interregional redistribution: fiscal equalization (FE) in the form of revenue sharing. Each region contributes a share $0<\beta<1$ of its gross revenues to the equalization system and receives half of the total contributions. The costs of tax enforcement, however, are still carried by the regions. Region $i$ 's net revenues then become

$$
R_{i}^{F E}=(1-\beta) R_{i}^{G}+\frac{\beta}{2} \sum R_{i}^{G}-c\left(p_{i}, \tilde{k}_{i}\right)
$$

where $R_{i}^{G} \equiv \tau_{i} \tilde{k}_{i}$ denotes the $g$ ross revenue. The uncoordinated, revenue maximizing choice is then characterized by

$$
\left(1-\frac{\beta}{2}\right)\left(\tau_{i} \frac{\partial k_{i}}{\partial p_{i}}+k_{i} \frac{\partial \tau_{i}}{\partial p_{i}}\right)-\frac{\beta}{2} \tau_{i} \frac{\partial k_{i}}{\partial p_{i}}+(1-\beta) 2 k_{i} \tau_{i} \frac{\partial \sigma_{i}}{\partial p_{i}}=C_{i}^{\prime} .
$$

Comparing (14) with the equivalent condition for the decentralized policy choice in a federal economy without any tax sharing (section 3), we get the following result:

Proposition 3 Under FE, the decentralized policy choice results in auditing rates which are below the level chosen in the absence of any tax sharing iff

(i) $\hat{\lambda}_{i}>0$ and $\hat{\mu}_{i}>\frac{1}{2}$.

The uncoordinated auditing rates under revenue sharing are above the level chosen in the absence of any tax sharing iff

(ii) $\hat{\lambda}_{i}>0$ and $\hat{\mu}_{i}<\frac{1}{2}$, or (iii) $\hat{\lambda}_{i}<0$.

Proof. See Appendix.

The overall impact from FE is similar - though, as we will show, not identical - to the one from FA, studied in the previous section. On the one hand, FE distorts the regions' incentives to enforce taxes as it reduces the marginal benefits from auditing without affecting marginal costs (first term in (14), the counterpart to effect A from section 4). On the other hand, the mechanism induces regions to partially internalize the externalities from auditing: it lowers the costs from an outflow of capital respectively HQs (second respectively third term in (14), similar to effect $\mathrm{B}$ respectively $\mathrm{C}$ from above). As long as $\hat{\lambda}_{i}<0$ (when the internalization of spillovers from effect $\mathrm{B}$ dominates the distortion), FE provides an unambiguous incentive to implement higher auditing rates as compared to the scenario without any interregional sharing respectively apportionment of revenues. If $\hat{\lambda}_{i}>0$ (when the distortion dominates effect $\mathrm{B}$ ) the 
overall impact from $\mathrm{FE}$ depends on $\hat{\mu}_{i}$, the strength of the internalization effect $\mathrm{C}$ relative to $\hat{\lambda}_{i}$, the sum from effect A and B. Whenever $\hat{\mu}_{i}<\frac{1}{2}$, the internalization effect $\mathrm{C}$ is sufficiently strong to outbalance - together with $\mathrm{B}$ - the distortionary effect. In this case, FE still works in favor of higher auditing rates. The opposite holds true for $\hat{\mu}_{i}>\frac{1}{2}$ (and $\hat{\lambda}_{i}>0$ ). Here, the competition mitigating effects are too weak to compensate the disincentives from FE.

Note that $\frac{1}{2}$ in the conditions for case (i) and (ii) in Proposition 3 can be interpreted as the marginal tax on a region's tax revenues, implicit in the equalization scheme. Under FE, regions contribute a fraction $\beta$ of their revenues and get back $\beta / n$, with $n$ denoting the number of jurisdictions within the economy. Allowing for $n>2$, the evaluation of (gross) revenue sharing would thus depend on the number of jurisdictions, as the distortionary effect is increasing respectively the internalization is decreasing - in $n$ (see Proposition 3 in Stöwhase and Traxler, 2005). Since the impact from the number of jurisdictions on the incentives from tax revenue sharing is well known (compare e.g. Köthenbürger, 2002), we focus on the case $n=2$.

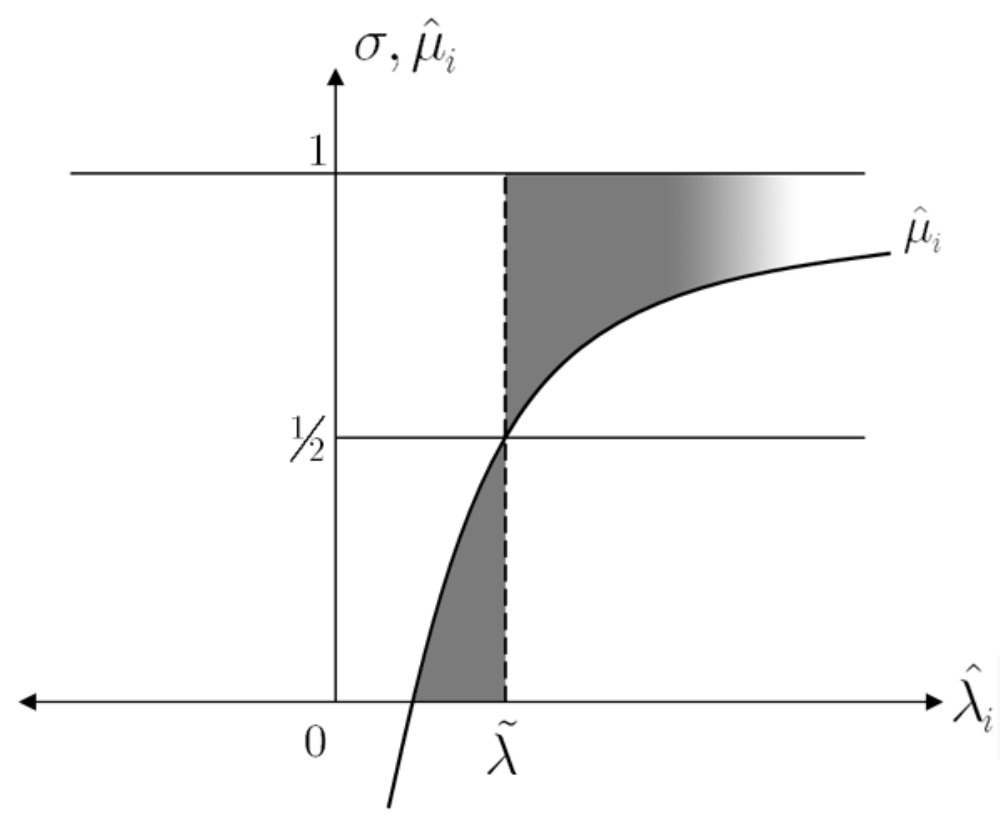

Figure 2: Impact of Fiscal Equalization

Figure 2 illustrates Proposition 3 graphically. The two areas where the introduction of FE would either increase or decrease auditing rates are separated by the dashed line at $\tilde{\lambda}_{i}$, with

$$
\tilde{\lambda}_{i} \equiv-4 k_{i} \tau_{i} \frac{\partial \sigma_{i}}{\partial p_{i}}>0
$$

corresponding to the level of $\hat{\lambda}_{i}$ for which $\hat{\mu}_{i}=\frac{1}{2} \cdot{ }^{22}$ For all $\hat{\lambda}_{i}<\tilde{\lambda}_{i}$, capital as well as HQ-mobility are sufficiently high, such that the alleviation of fiscal competition outweighs the distortion from revenue sharing. Contrarily, the disincentives dominate for $\hat{\lambda}_{i}>\tilde{\lambda}_{i}$.

\footnotetext{
${ }^{22}$ Note that $\tilde{\lambda}_{i}>0$ follows from $\partial \sigma_{i} / \partial p_{i}<0$, which is derived in (A.8) in the Appendix. (A.8) further implies that there always exists a finite value of $\hat{\lambda}_{i}$ for which revenue sharing has a negative impact on auditing efforts.
} 


\subsection{Qualitative Comparison of Apportionment and Fiscal Equalization}

A comparison of figures 1 and 2 respectively Propositions 2 and 3 shows that the impact of an apportionment and an equalization scheme are qualitatively equivalent for $\hat{\lambda}_{i}<0$. If $\hat{\lambda}_{i}>0$, however, the two schemes can work into different directions: For all $0 \leq \sigma_{i}<\hat{\mu}_{i} \leq \frac{1}{2}$ (illustrated by the grey shaded area to the left of $\tilde{\lambda}_{i}$ in figure 2), FA provides an incentive to reduce enforcement efforts, whereas FE would work in favor of more frequent auditing. The intuition behind this discrepancy is straightforward: While the incentives embedded in an apportionment system depend on the share of multiregional firms, this level of interregional integration does not play any role under FE. Whenever $0<\hat{\lambda}_{i}<\tilde{\lambda}_{i}$, the two mitigation effects dominate the distortion under FE - independently of $\sigma_{i}$. Under FA, however, the distortion can dominate for sufficiently high levels of interregional integration (if $\sigma_{i}<\hat{\mu}_{i}$ ).

The two different forms of tax sharing also have qualitatively different impacts for $\hat{\lambda}_{i}>\tilde{\lambda}_{i}$ whenever $\frac{1}{2} \leq \hat{\mu}_{i}<\sigma_{i} \leq 1$ (depicted by the second grey shaded area in figure 2). In this parameter range, capital as well as HQ-mobility are relatively low and the mitigation of fiscal competition has little impact. Under FE, the distortionary effect becomes dominant. Under FA, however, the distortion will be small if there are sufficiently few multiregional firms $\left(\sigma_{i}>\hat{\mu}_{i}\right)$. In this case, the two mitigation effects offset the distortion. Hence, FE would result in lower auditing efforts, whereas FA would trigger higher auditing rates. We summarize these findings in

Corollary 1 For $\hat{\lambda}_{i}>0$ the qualitative impact of a FA system on decentralized tax enforcement is different to the impact of FE iff either $0 \leq \sigma_{i}<\hat{\mu}_{i} \leq 1 / 2$ or $1 / 2 \leq \hat{\mu}_{i}<\sigma_{i} \leq 1$.

\subsection{Quantitative Comparison of Apportionment and Fiscal Equalization}

Let us now ask, under which of the two regimes - FA or FE - the decentralized policy choice will lead to stricter tax enforcement. The answer to this question is given by

Proposition 4 The decentralized policy choice under FA results in auditing rates which are below the level chosen under FE iff

(i) $\hat{\lambda}_{i}>0$ and $\zeta_{i}>\hat{\mu}_{i}\left[\zeta_{i}<\hat{\mu}_{i}\right]$, or

(ii) $\hat{\lambda}_{i}<0$ and $\zeta_{i}<\hat{\mu}_{i}\left[\zeta_{i}>\hat{\mu}_{i}\right]$

for $\alpha<\beta[\alpha>\beta]$ and

$$
\zeta_{i} \equiv \frac{\alpha \sigma_{i}-\beta / 2}{\alpha-\beta}
$$

Otherwise, the uncoordinated auditing rates under FA are above or, for the special case $\zeta_{i}=\hat{\mu}_{i}$, equal to the rates chosen under FE.

Proof. See Appendix. 
While the conditions of this proposition look slightly convoluted, they all derive from

$$
\left[\alpha\left(1-\sigma_{i}\right)-\beta / 2\right] \hat{\lambda}_{i}+(\alpha-\beta) 2 k_{i} \tau_{i} \frac{\partial \sigma_{i}}{\partial p_{i}}>0
$$

(see the Proof of Proposition 4 for the derivation). If condition (16) is fulfilled, the marginal benefits from auditing are higher under FE than under FA for any $p_{i}$. Replacing FA by a FE system would then trigger an increase in the auditing efforts.

Condition (16) simply compares the incentives from the two forms of sharing taxes. In particular, it compares the differences in the weighting of $\hat{\lambda}_{i}$ - the sum from the distortionary effect (A) and the mitigation of capital competition (B) - as well as the weighting of the mitigation of HQ-competition (effect C). To illustrate this, let us restrict to $\alpha<\beta$ and consider first scenario (i) from the Proposition. In this scenario $\hat{\lambda}_{i}>0$, i.e. the distortion from the redistribution of tax revenues dominates the mitigation effect B (for both schemes). The second term on the LHS of (16) is then unambiguously positive, reflecting that the mitigation effect $\mathrm{C}$ is larger under FE as $\beta>\alpha$ (remember that $\partial \sigma_{i} / \partial p_{i}<0$ ). Hence, it is sufficient for (16) to be satisfied, if the expression in the squared brackets is positive. Intuitively this is the case, whenever the distortion from FA - captured by the term $\alpha\left(1-\sigma_{i}\right)$ - is stronger than the one from FE. In contrast to this sufficient condition, $\zeta_{i}>\hat{\mu}_{i}$ represents the necessary (and sufficient) condition for (16) to hold under this scenario.

To see the intuition behind this latter condition, remember two points: First, note that $\hat{\mu}_{i}$ measures the degree of HQ-mobility (relative to $\hat{\lambda}_{i}$ ). The more sensitive the HQ location reacts to changes in the auditing rates, the lower $\hat{\mu}_{i}$ (for $\hat{\lambda}_{i}>0$ ), the easier it becomes for $\zeta_{i}>\hat{\mu}_{i}$ to hold. The reason behind this is that the mitigation effect $\mathrm{C}$ - which is larger under $\mathrm{FE}$ as $\beta>\alpha$-then becomes stronger, and the differences in the weights FA respectively FE put on $\hat{\lambda}_{i}$ are less crucial. Second, we know from our previous analysis that the distortion from FA will increase with a higher degree of interregional firm integration (a lower level of $\sigma_{i}$ ). This is reflected by $\partial \zeta_{i} / \partial \sigma_{i}<0$ (for $\alpha<\beta$ ), making $\zeta_{i}>\hat{\mu}_{i}$ more likely to hold for lower values of $\sigma_{i}$.

Scenario (ii) from Proposition 4 captures the case where $\hat{\lambda}_{i}<0$, i.e. where the mitigation effect $\mathrm{B}$ dominates the distortion (for both schemes). It would then be sufficient for (16) to hold, if the expression in the squared brackets is negative. This holds whenever the weight on $\hat{\lambda}_{i}$ under FA is smaller than under FE. In contrast to before, a high weighting now results in a stronger internalization effect, providing a more pronounced incentive to raise auditing rates. This is also reflected in the necessary (and sufficient) condition for this scenario, $\zeta_{i}<\hat{\mu}_{i}$, which is just the reversal of the condition for scenario (i).

\section{Joint System of Apportionment and Fiscal Equalization}

Finally we discuss the interaction of FA and FE in a tax sharing scheme which combines both elements of interregional redistribution. Motivated by the institutional arrangements in Germany, we consider a mechanism where revenues after apportionment are shared, whereas the costs of tax enforcement are still carried by the regions. A jurisdiction contributes a share $0<\beta<1$ of 
its gross revenues after apportionment, $R_{i}^{G A} \equiv R_{i}^{F A}+c\left(p_{i}, \tilde{k}_{i}\right)$, with $R_{i}^{F A}$ being defined in (11). Regional revenues net of enforcement costs are then given by

$$
R_{i}^{F F}=(1-\beta) R_{i}^{G A}+\frac{\beta}{2} \sum R_{i}^{G A}-c\left(p_{i}, \tilde{k}_{i}\right)
$$

The first order condition to the region's revenue maximization problem becomes

$$
\left(1-\gamma_{1}\right)\left(\tau_{i} \frac{\partial k_{i}}{\partial p_{i}}+k_{i} \frac{\partial \tau_{i}}{\partial p_{i}}\right)-\gamma_{1} \tau_{i} \frac{\partial k_{i}}{\partial p_{i}}+\gamma_{2} 2 k_{i} \tau_{i} \frac{\partial \sigma_{i}}{\partial p_{i}}=C_{i}^{\prime}
$$

where the terms

$$
\gamma_{1} \equiv \frac{\beta}{2}+\alpha(1-\beta)\left(1-\sigma_{i}\right) \geq 0 \quad \text { and } \quad \gamma_{2} \equiv(1-\beta)(1-\alpha) \geq 0
$$

capture the interaction of the incentives from FA and FE. A comparison with the decentralized auditing choice studied in section 3 leads us to

Proposition 5 Under a joint system of FA and FE the decentralized policy choice results in auditing rates which are below the level chosen in the absence of any tax sharing iff

(i) $\hat{\lambda}_{i}>0$ and $\delta_{i}<\hat{\mu}_{i}$.

The uncoordinated auditing rates will be above the level chosen in the absence of tax sharing iff

(ii) $\hat{\lambda}_{i}>0$ and $\delta_{i}>\hat{\mu}_{i}$, or (iii) $\hat{\lambda}_{i}<0$,

with

$$
\delta_{i} \equiv \frac{\beta / 2+\sigma_{i}(1-\beta) \alpha}{\beta+(1-\beta) \alpha}
$$

Proof. See Appendix.

As the joint tax sharing scheme represents a linear combination of FA and FE, its incentives are closely related to the respective isolated effects. Compared to an economy without any redistribution of tax revenues, the joint scheme provides incentives for higher auditing rates as long as $\hat{\lambda}_{i}<0$. This is not surprising, as the impact from FA and FE are qualitatively identical for this parameter range (compare Propositions 2 and 3 ).

More interesting is the case of $\hat{\lambda}_{i}>0$. For this parameter range, the interplay of FA and FE will result in stricter tax enforcement as compared to the benchmark scenario if $\delta_{i}>\hat{\mu}_{i}$. The opposite holds true for $\delta_{i}<\hat{\mu}_{i}$. It is straightforward that the joint effects from FA and FE tend to increase auditing rates, whenever $\mathrm{FA}$ and $\mathrm{FE}$ have the same effect. ${ }^{23}$

Remember that for $\hat{\lambda}_{i}>0$ the distortive effect from tax sharing (effect $\mathrm{A}$ ) is stronger than the mitigation of capital competition (effect B). Since the apportionment element within the joint scheme becomes less important with fewer multiregional firms (compare Proposition 2), the net

\footnotetext{
${ }^{23}$ One can easily show that $\sigma_{i}>\hat{\mu}_{i}$ and $1 / 2>\hat{\mu}_{i}$ together are sufficient for $\delta_{i}>\hat{\mu}_{i}$.
} 
distortion - the sum of effects $\mathrm{A}$ and $\mathrm{B}$ - is decreasing in $\sigma_{i}$. For a lower level of interregional integration (high values of $\sigma_{i}$ ), the mitigation of HQ-competition (effect $\mathrm{C}$ ) is therefore more likely to outbalance the distortion, and tax sharing would work in favor of stricter auditing. Formally, the condition $\delta_{i}>\hat{\mu}_{i}$ becomes less binding for high values of $\sigma_{i}$, since $\delta_{i}$ is increasing in $\sigma_{i}$.

What can we say about the impact of $\alpha$ and $\beta$ on the incentive from the joint tax sharing scheme? From Propositions 2 and 3 we know that the qualitative evaluation of the two isolated regimes are independent of the apportionment factor $\alpha$ and the level of equalization $\beta$ (as we do not consider income effects). In contrast, the qualitative impact from the joint scheme does depend on the level of $\alpha$ and $\beta$. The reason behind this is that the two layers of interregional redistribution may exert different incentives on decentralized tax enforcement for $\hat{\lambda}_{i}>0$ (see Corollary 1).

For $\sigma_{i}>\frac{1}{2}$, when the majority of firms maintain their HQs in the home region, one can derive from (18) that $\delta_{i}$ is increasing in $\alpha$ but decreasing in $\beta .{ }^{24}$ The intuition for this is the following: On the one hand, a higher apportionment factor only modestly increases the distortion when interregional firm integration is low (compare $\gamma_{1}$ in (17)). The incentive from FA to mitigate the HQ-competition (effect C), on the other hand, is unaffected by $\sigma_{i}$ (compare $\gamma_{2}$ in (17)). Hence, with $\sigma_{i}>\frac{1}{2}$ a higher apportionment factor $\alpha$ will cet.par. make it more likely that $\delta_{i}>\hat{\mu}_{i}$ holds, i.e. that the mitigation of the competition for HQs (effect $\mathrm{C}$ ) dominates the distortion.

An increase in the equalization factor $\beta$ has similar effects: (i) it raises the distortion, (ii) it mitigates the competition for mobile HQs and (iii) it tends to reduce the distortion from FA. While the first two effects are independent of $\sigma_{i}$, the third one - which works in favor of higher auditing rates - is not (compare $\gamma_{1}$ and $\gamma_{2}$ in (17)). The more firms retain their HQs at home, the less distortive is FA and the less crucial is the third, positive effect from FE. For $\sigma_{i}>\frac{1}{2}$ an increase in $\beta$ will then lower $\delta_{i}$, making it cet.par. harder for condition $\delta_{i}>\hat{\mu}_{i}$ to be fulfilled.

Following these arguments, it is clear that for a majority of multiregional firms $\left(\sigma_{i}<\frac{1}{2}\right)$, $\delta_{i}$ is decreasing in $\alpha$ but increasing in $\beta$. For this scenario, the distortion caused by FA is relatively strong. The joint tax sharing scheme is then more likely to increase decentralized auditing efforts, the smaller the apportionment and the larger the equalization factor. ${ }^{25}$

\subsection{Comparison of the Joint System and Fiscal Equalization}

Under which conditions will the joint tax sharing scheme provide incentives for stricter auditing as compared to a simple FE system? The answer to this question is given in

Proposition 6 The decentralized policy choice under a joint system of FA and FE results in auditing rates which are below the level chosen under a simple FE system iff

\footnotetext{
${ }^{24} \mathrm{An}$ extended appendix, which derives these (and further) results can be obtained from the author.

${ }^{25}$ Note that for the special case $\sigma_{i}=\frac{1}{2}$ we get $\delta_{i}=\frac{1}{2}$, independently of $\alpha$ and $\beta$. The impact of the joint system is therefore equivalent to the one of a simple $\mathrm{FE}$ scheme, studied in section 5 . The intuition behind this is that the two forms of tax sharing become equivalent if $\sigma_{i}=\frac{1}{2}$. This can also be seen by substituting $\sigma_{i}=\frac{1}{2}$ in (12) and comparing it with (14).
} 
(i) $\hat{\lambda}_{i}>0$ and $\sigma_{i}<\hat{\mu}_{i}$.

The uncoordinated auditing rates in a joint system are above the level chosen under FA iff

(ii) $\hat{\lambda}_{i}>0$ and $\sigma_{i}>\hat{\mu}_{i}$ or (iii) $\hat{\lambda}_{i}<0$.

Proof. See Appendix.

Introducing apportionment into a $\mathrm{FE}$ system has the same qualitative consequence on auditing efforts as the introduction of a pure FA system into an economy without any redistribution of tax revenues (compare Proposition 2). The intuition for this result is simply that FA interacts with $\mathrm{FE}$ in a linear way. The impact from a change in $\alpha$ on our three main effects from tax sharing - the distortionary effect (A), the mitigation of competition for mobile capital (B) respectively mobile HQs $(\mathrm{C})$ - is weighted by the same positive factor $(1-\beta)$. Hence, FA has the same qualitative consequences on decentralized tax enforcement, independently whether it is introduced as the only or as an additional layer of interregional redistribution together with FE. Whether or not it raises auditing rates solely depends on which effect - the distortion or the internalization - dominates. (Compare section 4.)

\subsection{Centralized Auditing Choice}

We conclude our analysis by asking how the decentralized enforcement policy under a joint system of FA and FE compares to the auditing level a central planer would establish. In our analysis, tax sharing is neutral with respect to total revenues in the economy. Hence, the problem of a central planer is equivalent to the one studied in section 3. Comparing the decentralized outcome from (17) with the centralized solution characterized in (10) yields the following result:

Proposition 7 The decentralized policy choice under a joint system of FA and FE results in auditing rates which are below the level a central planner would implement.

Proof. See Appendix.

The intuition behind the result is straightforward. Without any interregional redistribution, the competition for the mobile tax base results in auditing rates which are below the revenue maximizing levels. As tax sharing mitigates the fiscal competition, it tends to raise auditing rates and reduces the gap between the uncoordinated and the coordinated policy choice. Nevertheless, a system of FA and FE introduces a (further) distortion - regions carry the full costs of auditing but retain only a part of their collected revenues. While the distortionary effect does not necessarily dominate (compare Proposition 5), it prevents regions from implementing audit levels which are equal to (or even above) the ones chosen by a central authority.

Note, that the pure FA system studied in section 4 as well as the pure FE regime discussed in section 5 are both special cases of the joint system. Therefore, the result from Proposition 7 carries over for these two forms of tax sharing:

Corollary 2 The decentralized policy choice under a pure FA system $(\beta=0)$ as well as under a pure FE system $(\alpha=0)$ results in auditing rates which are below the level a central planner would implement. 


\section{Concluding Discussion}

This paper compares the incentives from formula apportionment (FA) and fiscal equalization (FE) on decentralized tax enforcement. Sub-national governments use their enforcement policies as strategic instruments to attract a mobile tax base. Within this context, both FA as well as FE, work as Pigouvian subsidies and mitigate the fiscal competition. At the same time, however, both regimes also distort the choice of enforcement efforts, as they redistribute tax revenues but leave the full enforcement costs to the regions. The tradeoff between these two opposing effects follows a similar, but in one aspect importantly different pattern under FA as compared to FE.

We discuss under which conditions which regime results in more effective tax enforcement. Our analysis highlights the role of the interregional firm integration - i.e. the share of multiregional companies - for the assessment of FA. We show that, for a sufficiently low level of interregional integration, FA will provide incentives for stricter tax enforcement, whereas a FE system can work into the opposite direction. Under the same conditions, the introduction of FA into an existing FE system would foster the effectiveness of tax enforcement. Hence, a FA cum FE regime could in principle work as an attractive tool, not only to redistribute tax revenues and counterbalance regional inequalities, but also to provide incentives for the decentralized choice of enforcement policies. This represents an important result for countries where institutional or political-economic constraints render a centralization of tax collection infeasible.

In order to provide a tractable and conclusive assessment of FA and FE, we employed several simplifying assumptions. While this seems to limit the scope of our results, we shall stress that the main properties of the different regimes would similarly turn up in a more general framework of $n>2$ asymmetric regions. In such a general model, however, the main incentives from FA and FE would be overlayed by other effects, some of which are already well studied in the fiscal competition literature. A nevertheless promising extension would be to study the endogenous choice of enforcement and tax policies. It would be interesting to see whether a welfare maximizing authority would counterbalance inefficiently low enforcement measures by higher statutory tax rates, thereby overly taxing those who fully comply with taxes.

Our framework may also serve as a benchmark for empirical research. Although some recent empirical contributions study the incentives from equalization transfers on decentralized fiscal policies (Büttner, 2006; Dahlby and Warren, 2003; Snoddon, 2003), the question of decentralized tax enforcement as well as the incentives related to apportionment have not been tackled so far. The only empirical work which considers an institutional setup similar to the one discussed here is Baretti et al. (2002). However, they neither have access to data on regional enforcement measures nor do they devote the necessary attention to the role of interregional firm integration - a factor which, as this paper has highlighted, exerts a central role in a transfer system that includes elements of apportionment. Future empirical evaluations of the incentives from any FA (cum FE) regime should account for this factor. 


\section{References}

Baretti, Christian, Bernd Huber and Karl Lichtblau (2002), A tax on tax revenue: The incentive effects of equalizing transfers: Evidence from Germany, International Tax and Public Finance 9, 631-649.

Black, Dan A. and William H. Hoyt (1989), Bidding for Firms, American Economic Review 79, 1249-1256.

Büttner, Thiess (2006), The Incentive Effect of Fiscal Equalization Transfers on Tax Policy, Journal of Public Economics 90, 477-497.

Cremer, Helmut and Firouz Gahvari (1993), Tax evasion and optimal commodity taxation, Journal of Public Economics 50, 261-275.

Cremer, Helmut and Firouz Gahvari (2000), Tax evasion, fiscal competition and economic integration, European Economic Review 44, 1633-1657.

Dahlby, Bev and Neil Warren (2003), The fiscal incentive effects of the Australian equalization system, Economic Record 79, 434-445.

Eggert, Wolfgang and Guttorm Schjelderup (2003), Symmetric tax competition under formula apportionment, Journal of Public Economic Theory 5, 439-446.

Jha, Raghbendra, M. S. Mohanty, Somnath Chatterjee and Puneet Chitkara (1999), Tax Efficiency in Selected Indian States, Empirical Economics 24(4), 641-654.

Klier, Thomas and William Testa (2002), Location trends of large company headquarters during the 1990s, Economic Perspectives, Federal Reserve Bank of Chicago, 12-26.

Knight, John and Shi Li (1999), Fiscal decentralization: Incentives, redistribution and reform in China, Oxford Development Studies 27, 5-32.

Köthenbürger, Marko (2002), Tax competition and fiscal equalization, International Tax and Public Finance 9, 391-408.

Libman, Alexander and Lars P. Feld (2007), Strategic Tax Collection and Fiscal Decentralisation: The Case of Russia, CESifo Working Paper No. 2031.

Martinez-Vazquez, Jorge and Andrey Timofeev (2005), Choosing between Centralized and Decentralized Models of Tax Administration, ISP Working Paper 05-02, Georgia State University.

Mikesell, John L. (2003), International Experiences with Administration of Local Taxes: A Review of Practices and Issues, Tax Policy and Administration Thematic Group, The World Bank, March 2003. 
Oates, Wallace (1999) An Essay on Fiscal federalism, Journal of Economic Literature 37(3), 1120-1149.

Pethig, Rüdiger and Andreas Wagener (2007), Profit Tax Competition and Formula Apportionment, International Tax and Public Finance 14, 631-656.

Riedel, Nadine and Marco Runkel (2007), Company tax reform with a water's edge, Journal of Public Economics 91, 1533-1554.

Sawicky, Max B. (2005), Do-It-Yourself-Tax-Cuts: The Crisis in U.S. Tax Enforcement, Economic Policy Institute Briefing Paper 160.

Senator fur Finanzen (2005), Die bremischen Ressorts im Städte- und Ländervergleich - BenchmarkingBericht 2005, Bremen.

Slemrod, Joel (2007), Cheating Ourselves: The Economics of Tax Evasion, Journal of Economic Perspectives 21(1), 25-48.

Smart, Michael (1998), Taxation and Deadweight Loss in a System of Intergovernmental Transfers, Canadian Journal of Economics 31(1), 189-206.

Snoddon, Tracy (2003), On Equalization and Incentives: An Empirical Assessment, Discussion Paper 2003-06EC, Laurier University, Waterloo.

Stöwhase, Sven and Christian Traxler (2005), Tax Evasion and Auditing in a Federal Economy, International Tax and Public Finance 12, 515-531.

Wildasin, David (1989), Interjurisdictional capital mobility: Fiscal externality and a corrective subsidy, Journal of Urban Economics 25, 193-212.

Wilson, John D. (1986), A Theory of Interregional Tax Competition, Journal of Urban Economics 19, 296-315.

Zodrow, George and Peter Mieszkowski (1986), Pigou, Tiebout, property taxation, and the underprovision of local public goods, Journal of Urban Economics 19, 356-370. 


\section{Appendix A. Comparative Static Results}

Applying the implicit function theorem on (2) respectively (3) we get

$$
\begin{gathered}
\frac{\partial e_{i}}{\partial p_{i}}=-\frac{s t}{g^{\prime \prime}}<0, \\
\frac{\partial e_{i}}{\partial t}=\frac{1-p_{i} s}{g^{\prime \prime}}>0 \\
\frac{\partial k_{i}}{\partial p_{i}}=\frac{s t e_{i}+\frac{\partial r}{\partial p_{i}}}{f^{\prime \prime}}<0 .
\end{gathered}
$$

From $\tau_{i} \equiv t\left(1-e_{i}+e_{i} p_{i} s\right)$ we can derive

$$
\frac{\partial \tau_{i}}{\partial p_{i}}=s t\left(e_{i}+\frac{t\left(1-p_{i} s\right)}{g^{\prime \prime}}\right)>0
$$

where we made use of (A.1) and $p_{i} s<1$. Further differentiating (A.4) then gives

$$
\frac{\partial^{2} \tau_{i}}{\partial p_{i}^{2}}=-\frac{(s t)^{2}}{g^{\prime \prime}}\left(2-\frac{t g^{\prime \prime \prime}\left(1-p_{i} s\right)}{\left(g^{\prime \prime}\right)^{2}}\right)<0,
$$

where we assume that the first order effect dominates.

As $\partial \pi_{j} / \partial p_{i}=0$, it follows from (5) respectively (6) that

$$
\frac{\partial \sigma_{i}}{\partial p_{i}}=h\left(\hat{\theta}_{i}\right) \frac{\partial \pi_{i}}{\partial p_{i}}
$$

Differentiating the profits from (1) yields

$$
\frac{\partial \pi_{i}}{\partial p_{i}}=\frac{\partial k_{i}}{\partial p_{i}}\left[f^{\prime}\left(k_{i}\right)-\left(r+g\left(e_{i}\right)+\tau_{i}\right)\right]-\frac{\partial e_{i}}{\partial p_{i}} k_{i}\left[g^{\prime}\left(e_{i}\right)+\frac{\partial \tau_{i}}{\partial e_{i}}\right]-\frac{\partial \tau_{i}}{\partial p_{i}} k_{i} .
$$

Using (2) and (3) one can easily show that the expressions in both squared brackets must equal zero for the firm's optimal choice. Hence, we finally get

$$
\frac{\partial \sigma_{i}}{\partial p_{i}}=-h\left(\hat{\theta}_{i}\right) \frac{\partial \tau_{i}}{\partial p_{i}} k_{i}<0
$$

which is strictly negative (and finite) due to (A.4) and our assumption on $h($.$) . Following the$ same steps as above, it is easy to show $\partial \sigma_{i} / \partial p_{i}=-\partial \sigma_{j} / \partial p_{i}$.

Finally, we derive from the definition of $\hat{k}_{i}$ and (4)

$$
\frac{\partial \tilde{k}_{i}}{\partial p_{i}}=\frac{\partial k_{i}}{\partial p_{i}}\left(1+\sigma_{i}-\sigma_{j}\right)+k_{i}\left(\frac{\partial \sigma_{i}}{\partial p_{i}}-\frac{\partial \sigma_{j}}{\partial p_{i}}\right)<0,
$$

where (A.3) and (A.8) imply that the whole term must be negative. 


\section{Appendix B. Proofs}

Proof of Proposition 1. Rearranging (8) yields

$$
\tilde{k}_{i} \frac{\partial \tau_{i}}{\partial p_{i}}+\left(\tau_{i}-c_{2}\right) \frac{\partial \tilde{k}_{i}}{\partial p_{i}}=c_{1}
$$

Note that in a plausible equilibrium there must hold $\tau_{i}>c_{2}$ - otherwise a region could increase its net revenues by reducing the tax base. As $\partial \tilde{k}_{i} / \partial p_{i}<0$, the LHS in this condition is lower than the LHS in (10) for any $p_{i}$.

Proof of Proposition 2. From (11) we get

$$
\begin{gathered}
\sigma_{i}\left(k_{i i} \frac{\partial \tau_{i}}{\partial p_{i}}+\tau_{i} \frac{\partial k_{i i}}{\partial p_{i}}\right)+\alpha\left(1-\sigma_{i}\right) \frac{\partial k_{i j}}{\partial p_{i}} \tau_{j}+(1-\alpha)\left(1-\sigma_{j}\right)\left(k_{j i} \frac{\partial \tau_{i}}{\partial p_{i}}+\tau_{i} \frac{\partial k_{j i}}{\partial p_{i}}\right) \\
+\frac{\partial \sigma_{i}}{\partial p_{i}}\left(k_{i i} \tau_{i}-\alpha k_{i j} \tau_{j}\right)-\frac{\partial \sigma_{j}}{\partial p_{i}}(1-\alpha) k_{j i} \tau_{i}=C_{i}^{\prime}
\end{gathered}
$$

Making use of

$$
\frac{\partial k_{j i}}{\partial p_{i}}=\frac{\partial k_{i i}}{\partial p_{i}} \equiv \frac{\partial k_{i}}{\partial p_{i}}
$$

as well as (4) and the fact that

$$
\sigma_{i}=\sigma_{j}, \quad k_{i}=k_{j}, \quad \tau_{i}=\tau_{j}, \quad \frac{\partial k_{i}}{\partial p_{i}}=-\frac{\partial k_{j}}{\partial p_{i}}, \quad \frac{\partial \sigma_{i}}{\partial p_{i}}=-\frac{\partial \sigma_{j}}{\partial p_{i}}
$$

holds in a symmetric equilibrium, we arrive after some rearranging at (12).

Using (A.9) together with $\sigma_{i}=\sigma_{j}$ in (8), we can rewrite the first-order condition as

$$
\tau_{i} \frac{\partial k_{i}}{\partial p_{i}}+k_{i} \frac{\partial \tau_{i}}{\partial p_{i}}+2 k_{i} \tau_{i} \frac{\partial \sigma_{i}}{\partial p_{i}}=C_{i}^{\prime}
$$

Since we consider symmetric equilibria between perfectly symmetric region together with an exogenous capital supply, the equilibrium capital allocation will be the same across different scenarios. The RHS of the different first order conditions will therefore be the same for any $p_{i}$, and we only have to consider the marginal benefits from auditing. (This argument is also applied in the following proofs.)

The comparison with condition (12) then shows, that the LHS in (A.12) is strictly larger than the LHS in (12) if

$$
\alpha\left(1-\sigma_{i}\right)\left(k_{i} \frac{\partial \tau_{i}}{\partial p_{i}}+2 \tau_{i} \frac{\partial k_{i}}{\partial p_{i}}\right)+\alpha 2 k_{i} \tau_{i} \frac{\partial \sigma_{i}}{\partial p_{i}}>0 .
$$


Simplifying and rearranging we can rewrite this inequality as

$$
\begin{array}{ccc}
\sigma_{i}<1+2 k_{i} \tau_{i} \frac{\partial \sigma_{i}}{\partial p_{i}} \frac{1}{\hat{\lambda}_{i}} & \text { if } \quad \hat{\lambda}_{i}>0 \\
\sigma_{i}>1+2 k_{i} \tau_{i} \frac{\partial \sigma_{i}}{\partial p_{i}} \frac{1}{\hat{\lambda}_{i}} & \text { if } & \hat{\lambda}_{i}<0
\end{array}
$$

Since $\partial \sigma_{i} / \partial p_{i}<0$, the RHS of the inequality - which corresponds to $\hat{\mu}_{i}$ from (13) - is smaller than unity as long as $\hat{\lambda}_{i}<0$. Hence, for any $p_{i}$ the marginal benefits of auditing in the case without FA are higher [lower] as in the scenario with FA if (i) $\hat{\lambda}_{i}<0$ and $\sigma_{i}<\hat{\mu}_{i}$ [(ii) if $\hat{\lambda}_{i}<0$ and $\left.\sigma_{i}>\hat{\mu}_{i}\right]$. For the case with $\hat{\lambda}_{i}<0$, the RHS of the inequality is strictly greater than one. As $\sigma_{i} \in[0,1]$, the second condition can then never hold. Therefore, the marginal benefits of auditing without FA are always below the marginal benefits under FA as long as (iii) $\hat{\lambda}_{i}<0$.

Proof of Proposition 3 and 5. The first order condition to the regions' problem under the joint system of FA and FE,

$$
\max _{p_{i}} R_{i}^{F F}
$$

is given by

$$
\begin{aligned}
& (1-\beta)\left\{\left(1-\alpha\left(1-\sigma_{i}\right)\right)\left[\tau_{i} \frac{\partial k_{i}}{\partial p_{i}}+k_{i} \frac{\partial \tau_{i}}{\partial p_{i}}\right]-\alpha\left(1-\sigma_{i}\right) \tau_{i} \frac{\partial k_{i}}{\partial p_{i}}+2 k_{i} \tau_{i}(1-\alpha) \frac{\partial \sigma_{i}}{\partial p_{i}}\right\} \\
& +\frac{\beta}{2}\left\{\left[\tau_{i} \frac{\partial k_{i}}{\partial p_{i}}+k_{i} \frac{\partial \tau_{i}}{\partial p_{i}}\right]+\tau_{j} \frac{\partial k_{j}}{\partial p_{i}}\right\}=C_{i}^{\prime}
\end{aligned}
$$

Rearranging and substituting for (4) and (A.11), we arrive at (17). Comparing the LHS of (17) and (8), the former is strictly greater than the latter if

$$
-\gamma_{1} \hat{\lambda}_{i}-\left(1-\gamma_{2}\right) 2 k_{i} \tau_{i} \frac{\partial \sigma_{i}}{\partial p_{i}}>0
$$

with $\gamma_{1}$ respectively $\gamma_{2}$ as defined in the main text and $\gamma_{2}<1$. After some rearranging, we can rewrite this condition as

$$
\begin{array}{lll}
\delta_{i}>\hat{\mu}_{i} & \text { if } & \hat{\lambda}_{i}>0 \\
\delta_{i}<\hat{\mu}_{i} & \text { if } & \hat{\lambda}_{i}<0
\end{array}
$$

with $\delta_{i}$ as defined in (18). From the Proof of Proposition 2 we know that $\hat{\mu}_{i}>1$ for $\hat{\lambda}_{i}<0$ (assuming $\hat{\lambda}_{i} \neq-\infty$ ). Since $0 \leq \delta_{i} \leq 1$, condition (A.17) is then always fulfilled for $\hat{\lambda}_{i}<0$. This proves Proposition 5.

From this result immediately follows Proposition 3. First, note that $\delta_{i}=\frac{1}{2}$ for $\alpha=0$. Condition (A.17) then simplifies to

$$
\begin{array}{lll}
\frac{1}{2}>\hat{\mu}_{i} & \text { if } \quad \hat{\lambda}_{i}>0 \\
\frac{1}{2}<\hat{\mu}_{i} & \text { if } & \hat{\lambda}_{i}<0
\end{array}
$$

which proves Proposition 3. 
Proof of Proposition 4. Comparing the LHS of condition (12) with the one in condition (14), the latter is strictly smaller than the former if

$$
\hat{\lambda}_{i}\left(\alpha\left(1-\sigma_{i}\right)-\frac{\beta}{2}\right) \geq(\beta-\alpha) 2 k_{i} \tau_{i} \frac{\partial \sigma_{i}}{\partial p_{i}} .
$$

Rearranging and substituting for $\hat{\mu}_{i}$ and $\zeta_{i}$ as defined in the main text, we arrive at

$$
\begin{array}{ll}
\zeta_{i} \geq \hat{\mu}_{i}\left[\zeta_{i} \leq \hat{\mu}_{i}\right] & \text { if } \quad \hat{\lambda}_{i}>0 \text { and } \alpha<\beta[\alpha>\beta] \\
\zeta_{i} \geq \hat{\mu}_{i}\left[\zeta_{i} \leq \hat{\mu}_{i}\right] & \text { if } \quad \hat{\lambda}_{i}<0 \text { and } \alpha>\beta[\alpha<\beta]
\end{array}
$$

where $\alpha \neq \beta$ has to hold. If the respective inequalities between $\zeta_{i}$ and $\hat{\mu}_{i}$ hold strictly, auditing rates under FA will be below the levels chosen under FE. For the special case where $\zeta_{i}=\hat{\mu}_{i}$, the two schemes lead to the same auditing rates. Otherwise, if the inequalities are violated, auditing efforts will be higher under FA than under a FE system.

Proof of Proposition 6. Comparing the LHS of condition (12) with the one in condition (17), the latter is strictly greater than the former if

$$
-\alpha(1-\beta)\left(1-\sigma_{i}\right) \hat{\lambda}_{i}>-\alpha(1-\beta) 2 k_{i} \tau_{i} \frac{\partial \sigma_{i}}{\partial p_{i}} .
$$

With $\alpha>0$ and $\beta<1$ we can rewrite this inequality as

$$
\begin{array}{lll}
\sigma_{i}>\hat{\mu}_{i} & \text { if } & \hat{\lambda}_{i}>0 \\
\sigma_{i}<\hat{\mu}_{i} & \text { if } & \hat{\lambda}_{i}<0,
\end{array}
$$

where we have substituted for $\hat{\mu}_{i}$. Remember that for $\hat{\lambda}_{i}<0$ there holds $\hat{\mu}_{i}>1$. As $\sigma_{i} \in[0,1]$ the inequality is always fulfilled for $\hat{\lambda}_{i}<0$.

Proof of Proposition 7. Substituting for $C_{i}^{\prime}$ and (A.9), we can rewrite (17) as

$$
\left(1-\gamma_{1}\right)\left(\tau_{i} \frac{\partial k_{i}}{\partial p_{i}}+k_{i} \frac{\partial \tau_{i}}{\partial p_{i}}\right)-\gamma_{1} \tau_{i} \frac{\partial k_{i}}{\partial p_{i}}+\gamma_{2} 2 k_{i} \tau_{i} \frac{\partial \sigma_{i}}{\partial p_{i}}-c_{2}\left(\frac{\partial k_{i}}{\partial p_{i}}+2 k_{i} \frac{\partial \sigma_{i}}{\partial p_{i}}\right)=c_{1}
$$

Note further, that the problem of a central authority under a joint system of FA and FE is equivalent to the case without any interregional redistribution of revenues. The comparison of the above condition with (10) then shows that - for any $p_{i}$ - the central authority considers higher marginal benefits from auditing as the regions do under the joint tax sharing scheme if

$$
-\gamma_{1} k_{i} \frac{\partial \tau_{i}}{\partial p_{i}}+\tau_{i} \frac{\partial k_{i}}{\partial p_{i}}\left[\tau_{i}\left(1-2 \gamma_{1}\right)-c_{2}\right]+2 k_{i} \frac{\partial \sigma_{i}}{\partial p_{i}}\left[\tau_{i} \gamma_{b}-c_{2}\right]<0 .
$$

For any reasonable equilibrium, the expressions in the squared brackets in the second and third term on the LHS must be positive. Otherwise a region could increase net revenues by pushing out capital respectively HQs from the economy. Since $\gamma_{1} \geq 0$, the LHS is unambiguously negative and the condition is always fulfilled. 